\title{
SMART GROCERY MANAGEMENT SYSTEM USING INTERNET OF THINGS
}

\author{
Chetal. S. Patil ${ }^{1}$, Kanaksing. N. Pawar ${ }^{2}$ \\ ${ }^{1}$ ME Final Year Student, Electronics Department, SSVPS COE, Dhule, (India) \\ ${ }^{2}$ Head of Electronics Department, SSVPS COE Dhule, (India)
}

\begin{abstract}
Developing smart phones and smart systems can provide a different dimension to the method of our shopping, banking, and several activities that we carry out in our day to day life. Smart grocery system is a smart and new way of grocery shopping that acts as our helper and re-defines the approach towards grocery shopping. For an oversized range of various heterogeneous end systems, the internet of Things (IOT) could be ready to incorporate seamlessly and transparently. Whereas it also provides open access to choose subsets of information for an event of digital services. Therefore building a general design for the IoT is really a advanced task, in the main attributable to the extraordinarily massive kind of services, devices and link layer technologies, which will be concerned in such a system. For the smart city application we specifically focus on IOT, in this system. This system helps us to maintain the smart grocery management which is basic requirement for every household person. Here we are going to study the level measurement sensing using ultrasonic sensor of solid or liquid substances as well as temperature measurement using temperature sensor, and according to that the order will be placed for shopping of that particular grocery. This system introduces us to the simple implementation and usable application with the low cost solution to the common man.
\end{abstract}

Keywords- open access, link layer technologies, heterogeneous end system.

$* * *$

\section{INTRODUCTION}

Internet of Everything or Network of Everything is additionally known as Internet of Things (IoT). When physical objects or things are embedded with physics, sensors and software then the network called IOT is formed. This network has property to change objects to exchange knowledge with the assembly, operator and/or different connected devices supported the infrastructure of International Telecommunication Union's international Standards Initiative. IOT permits the detection of objects and controlling them remotely across existing network infrastructure. Thus a network makes a scope for a lot of direct integration between the physical world and computerbased systems. This leads to improved accuracy, potency and economic profit. Every factor is unambiguously acknowledgeable through its embedded system however is in a position to interoperate among the present internet infrastructure.

Now it is possible to visualizes a near future with the recent communication paradigm that is Internet of Things (IoT), where the thing of daily life are going to be equipped with transceivers, microcontrollers, for electronic communication between the things and create them ready to communicate with each other and with the users [1]. Thus the IoT is a thought of creating the network which sanctions quick access and interaction with a large form of devices like, home appliances, police investigation cameras, observation sensors, displays, actuators, vehicles, and so on. The variety of applications of IOT can create use of the possibly huge quantity and kind of information generated by such objects for supplying new services to companies, public administrations and citizens. IOT has applications in various domains, such as industrial automation, home automation, mobile care, medical aids intelligent energy management and traffic management, smart grid and plenty of others. [2].

An application that has been designed for future generation smart shopping system is called Smart Grocery System. The name itself suggests the smartness of the system. This system will help the user to get proper management of grocery in our kitchen. The user no longer has to worry about continuously monitoring groceries at our homes. Here we get notification when the level of content in the storage bottles will be low, then immediately we can place order for that particular content. Level measurement is the continuous task going on here by level measurement sensor .As well as simultaneously this system will give us idea about the temperature inside the bottle so that protecting the content according to the weather conditions, and suitable temperature can be maintain for different content as per its requirement. Temperature is measured using temperature sensor.

Section 2 provides an outline of the problems we face. Section 3 describes the proposed solution to the problem and section 4. Gives measuring principle and operation mode of smart grocery system. The paper is concluded with a brief discussion of the benefits of system to society for creating our city smart. 


\section{PROBLEM STATEMENT}

Grocery shopping these days has become a job. The client needs to continuously monitor groceries at home and also has the work of managing coupons, maintaining shopping lists, standing in restraint out queues, reading the fine print on food cans, and even needs to find out within which rack and row he or she may notice that item. A big proportion of the grocery shoppers would thus have an interest in an additional convenient, quicker grocery shopping option. Now a day's life for everyone has become so hectic and time consuming, at such time we require a smart system at our kitchen also. To put on records and observing all the grocery at home is difficult. Most of the time we remain in wrong belief that we have enough grocery in our kitchen but we have to face empty bottles at the time of emergency when the requirement is must that gives us inconvenience. And to avoid this, some time we buy more than enough grocery \& store it at our home for many days, which is also an inconvenience can cause damage to grocery. Both this situations are problems. System that can give continuous level measurement and can notify us about low level of content is required to avoid these problems. Further in section 3 we will describe details of this solution

\section{PROPOSED SOLUTION - SMART GROCERY}

\section{SYSTEM}

This plugin uses sensing and inbuilt Wi-Fi capabilities of the Edison board to connect to cloud. Smart City plugin is cloud enabled solution which helps in remote monitoring, alerting and decision making. Smart City Plugin android app helps in mobile monitoring and alerting on-the-go. This facilitates us with smart system of shopping the grocery. This system intimate user when the levels of groceries are low. It gives the live details of the levels of grocery in the container also it intimates the temperature and humidity of the content so we can also determine if it is in good condition or not.
The SMART Grocery management can provide benefits as follows:

- Automated ordering to grocery stores - on daily/ weekly/ monthly basis.

- The consumption pattern_of the different foods based on which the user can be provided inputs on how much more / less he can place order for a particular item.

\section{SYSTEM DEVELOPMENT \\ REQUIREMENTS}

AND

Smart grocery system was developed using Intel Edison Ardunio board. Here HC-SR04 Ultrasonic sensor is used for level measurement which gives the signal of low level to the board. Temperature sensor is used to measure the humidity in the container. And messages are sending by MQTT protocol for communication to the cloud connectivity.

\subsection{System Requirements}

Below hardware and software requirements are in detail.

\section{Hardware Requirement}

1 Intel Edison board/Ardunio uno board (5V, 8-bit, $16 \mathrm{MHz}$ AVR)

2 Ultrasonic sensor (HC-SR04)

3 Temperature sensor

\section{Software Requirement}

1. Node.js (Intel XDK IDE) for Cloud connectivity

2. Arduino IDE for sensor detection

3. Eclipse IDE for Android application development

4. IBM Bluemix PaaS cloud

5. MQTT protocol for communication

\subsection{System Architecture}

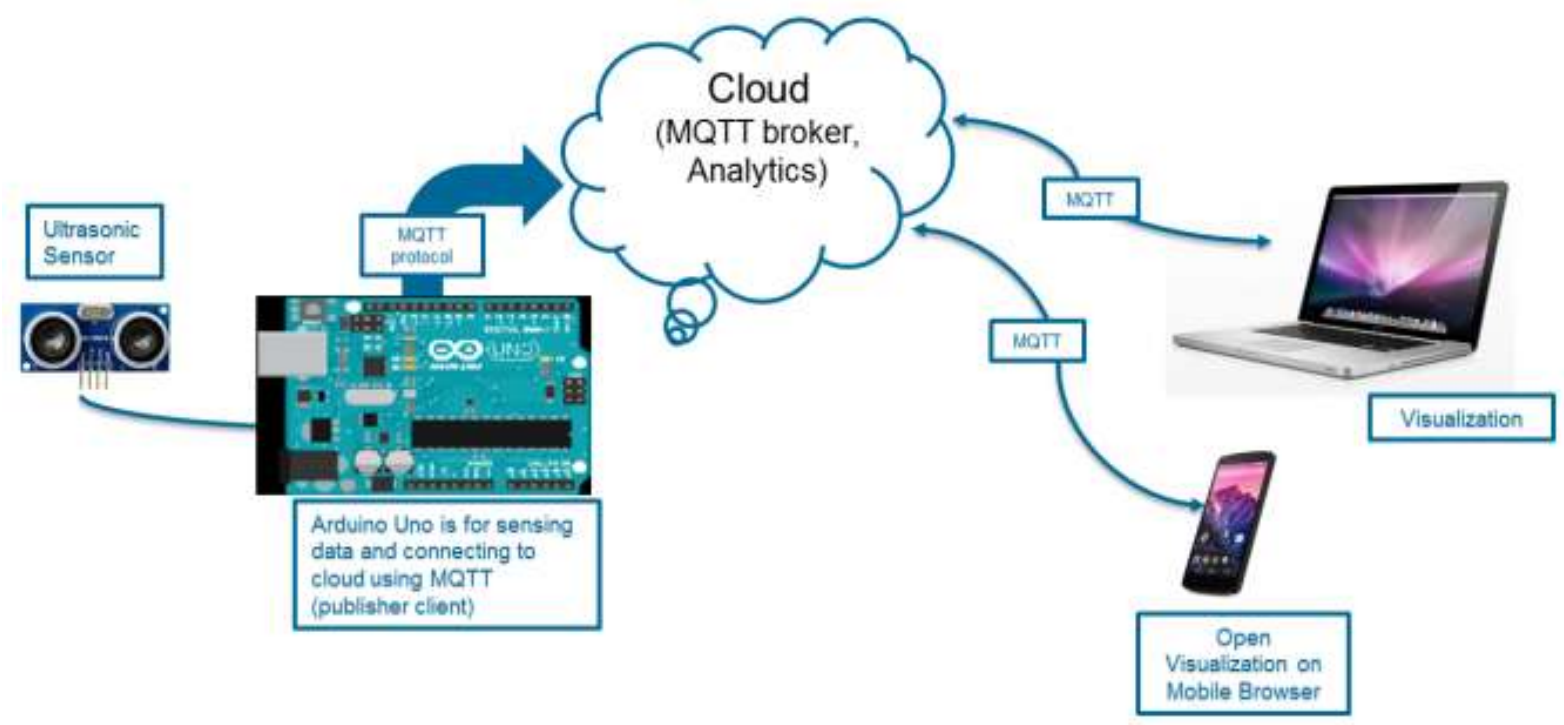

Fig 1. Block diagram of smart grocery system 


\section{Diagram Description}

Ultrasonic sensor: Ultrasonic sensors can both sense and transmit hence they are also called as transceivers. Ultrasonic sensors works on a principle as same as that of transducers, transducers convert ultrasound waves to electrical signals or contrariwise. They can solve even the most advanced tasks involving object detection or level measurement. When ultrasonic sensing elements generate high-frequency sound waves then an echo is generated that is received back by the sensor. The distance to an object is measured by measuring the time interval between sending the signal and receiving the echo. Once the ultrasonic wave is launched, the timing is begun and ultrasonic transmitter emits an ultrasonic wave in one direction. As soon as this wave which is spreaded in air will encountered an obstacles on the way would come back instantly. Once the ultrasonic receiver would receive the reflected wave it will stop timing.

\section{Operation Mode}

The period of time taken by ultrasonic pulses to reach to the surface of the medium and back is used to determine continuous level measurement. The modified angle during filling and emptying granulated solids and also the rough liquid surfaces influence the reflection of the ultrasonic pulse. This will also impact the measurement of Chemical and physical properties of the medium don't influence the measurement result. Hence the abrasive and aggressive, viscous and adhesive media will be easily measured.

\section{Measuring Principle}

The principle of measuring ultrasonic distance is used here for measuring the time from launch to reflection. Once the obstacle is encountered, and then the distance between the transmitter and obstacle is calculated .The calculation done according to the time and also the speed, and also according to time difference distance measurement principle. This measured distance output is send to Arduino uno board

Arduino Uno: Arduino is an open-source platform. That is incredibly easy-to-use for hardware and software. Arduino boards can read inputs like light on a sensor, a finger on a button, and switch it into an output - activating a motor, turning on an LED. All this can be defined by a group of instructions programmed through the Arduino software (IDE). Arduino was born at the Ivrea Interaction design Institute as a simple tool for quick prototyping. This board differs from simple 8-bit boards as they are used for IoT applications and embedded environments. Arduino Uno is a microcontroller board based on the ATmega328P.The output from ultrasonic sensor is connected to the board, and the board is connected to a computer with a USB cable this board is used for sensing data and connecting it to cloud using MQTT protocol (publisher client).

MQTT Protocol: Need to manage the communication between these connected devices is a focus of the Internet of
Things. Most web traffic is carried using a protocol called Hypertext Transfer Protocol (HTTP). But HTTP has a number of limitations that make it a poor choice as an Internet of Things protocol. As MQTT offers a number of significant advantages over HTTP such as improved user experience, more flexibility and scalability, lower running costs, lower development costs and insanely efficient, hence MQTT protocol is used for internet of things.

MQTT is Message Queue telemetry Transport it is a publish/subscribe protocol, which enables easy broadcasting of messages from one publisher to several subscribers. As shown in fig 1.By sending a message to the broker the connection is initiated through a publisher. Once the message is received the broker provides response to that with a status code. MQTT clients are behind routers. To translate from a private network address (like 192.168.x.x, 10.0.x.x) to a public they use network address translation (NAT). After establishing the connection the broker has to keep it open as long as the publisher doesn't send a disconnect command or it loses the connection. It is very simple and lightweight messaging protocol, with lowbandwidth, unreliable networks or high-latency. The principle of design aims to reduce network bandwidth and device resource needs. MQTT provides a scalable and cost effective way to connect your devices over the internet. MQTT additionally provides security by permitting to send a username and password for authenticating the client and also authorization. Then as shown in fig, we can get open visualization on our mobile or computer through the cloud.

\section{ALGORITHM/STEPS}

- Attaché the ultrasonic sensor to Ardunio board. Here we are using four GPIO pins of Ardunio uno - vcc, ground, trigger and echo.

- Calculate distance by using trigger and echo pin of ultrasonic sensor.

- Send/publish this distance data by using PubSubclient library by using Jason format and MQTT protocol to cloud server.

- Get the data at cloud server and visualize the data in the form of graph by using visualizer. As per the data make the decision_whether we want to order the product or not.

\section{RESULTS}

The data sensed by the sensor is given to the board and the USB connection from the board is given to computer. As shown in fig 2. (a) And 2. (b) The output data is displayed on serial monitor, through the cloud using MQTT protocol. Fig 2.(a) shows the network connection by initially running DTPH test program and identifying IP address 192.168.1.3, it also shows initialization of sensor, client id, device name and MQTT broker name. All the initialization prints are displayed as soon as the connection has established. 
COM4 (Arduino/Genuino Uno)

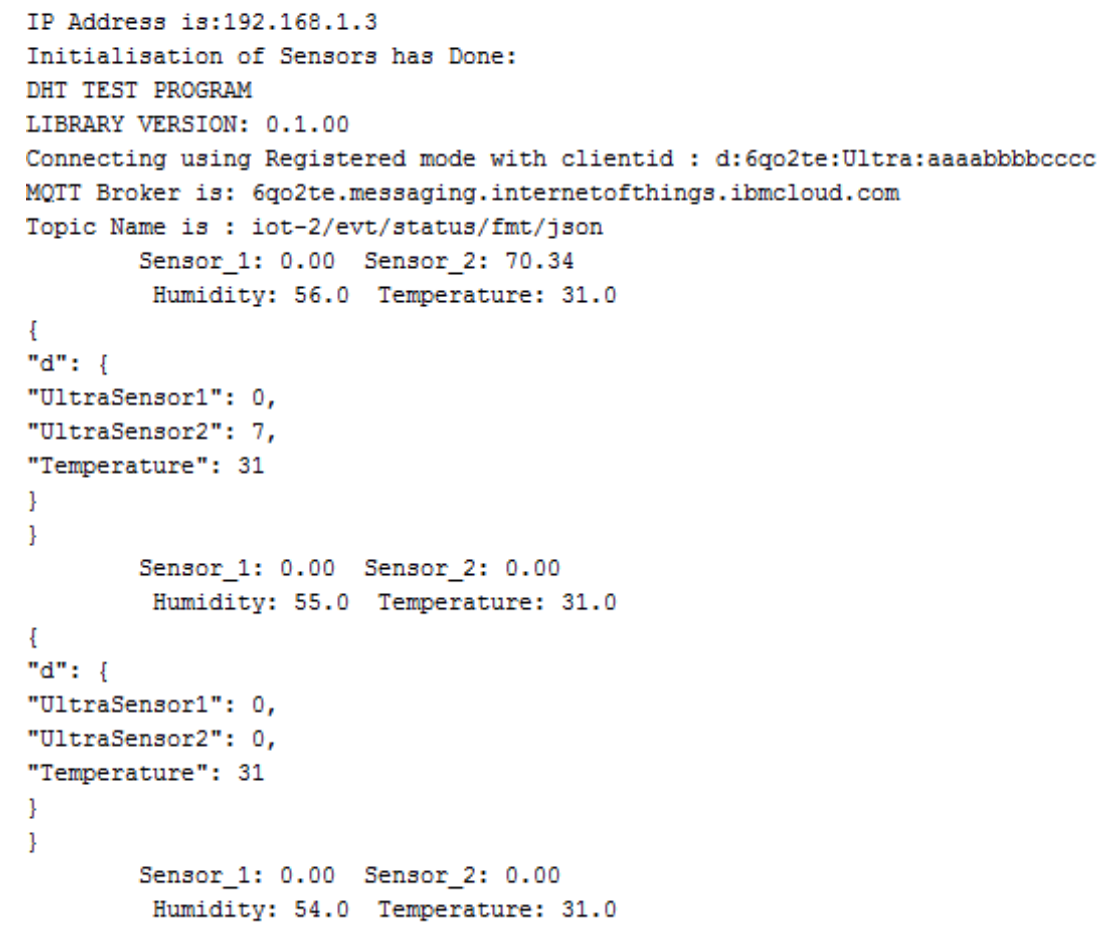

Fig 2 (a) IP Address detection and initialization of sensors

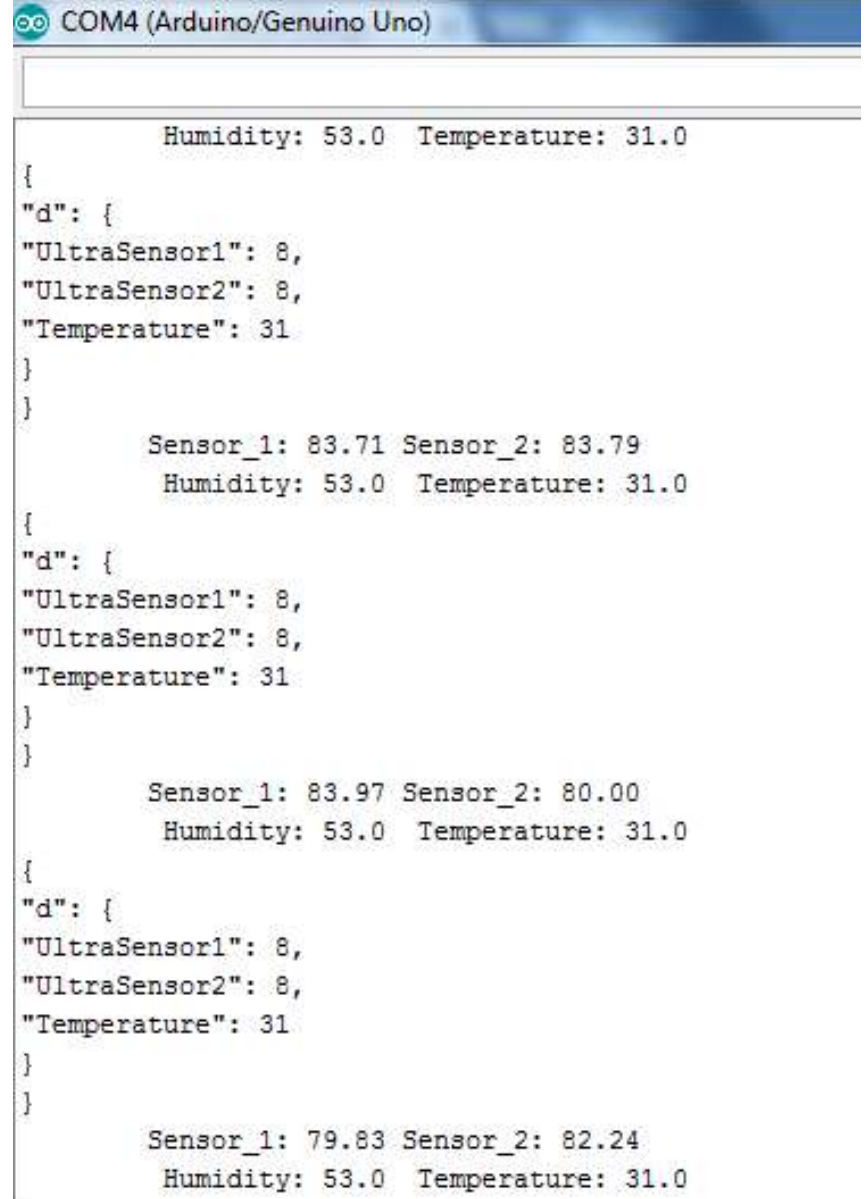

Fig 2 (b) Changes in values of sensor1, sensor2, temperature, humidity
Fig 2. (b) Shows change in values of sensors as well as temperature and humidity. The live and instant changes in values of sensor 1 and sensor 2 are shown at output according to the change in distance measured by sensors. Both the sensors are attached to different container, they sense the level of the grocery in the container and gives the graphical output. According to that output we can decide whether we want to place the order for that item or not. In this way we can get the notification of our groceries and we can place the order for it according to our choice

\section{CONCLUSION}

In this paper, we have done the analysis of the solutions available for the implementation of smart grocery system using IoTs. Smart grocery system using IoT is cost effective and user friendly system for customers. It not only helps to induce organized for grocery shopping, it additionally saves our time and money. With smart grocery system, one can be relax and stop worrying of continuously being in follow, and checking the grocery containers in our house. We will get notification about the low level of grocery and we can place order for particular item. This helps in ease of society and one step forward of making our city smart.

Future work or related work for smart grocery system is we can directly place order by android application in our smart phones, so developing an application to automatically placing order to grocery shops is further implementation in this system 


\section{ACKNOWLEDGEMENT}

I would like to thank my guide, Prof. K.N.Pawar and staff of electronics department for their guidance and precious time allotted me for this work. Also a heartily thank to SSVPS COE, Dhule for valuable inputs and directions for shaping this project.

\section{REFERENCES}

[1]. L. Atzori, A. Iera, and G. Morabito, "The internet of things: A survey," Comput. Netw., vol. 54, no. 15, pp. 2787-2805, 2010.

[2]. P. Bellavista, G. Cardone, A. Corradi, and L. Foschini, "Convergence of MANET and WSN in IoT urban scenarios," IEEE Sens. J., vol. 13, no. 10,pp. 3558-3567, Oct. 2013.

[3]. Andrea Zanella, Senior Member, IEEE, Nicola Bui, Angelo Castellani, Lorenzo Vangelista, Senior Member, IEEE, and Michele Zorzi, Fellow, IEEE "Internet of Things for Smart Cities", Ieee Internet Of Things Journal, Vol. 1, No. 1, February 2014.

[4]. Sangeetha shekar, prashant Nair and abdelsalam (sumi) Helel "iGrocer- A Ubiquitous and Pervasive Smart Grocery Shopping System University of Florida, Gainesville, FL32611, USA

[5]. M. Dohler, I. Vilajosana, X. Vilajosana, and J. Llosa, "Smart Cities: An action plan," in Proc. Barcelona Smart Cities Congress, Barcelona, Spain, Dec. 2011, pp. 1-6.

[6]. Kary Fr"amling, Sylvain Kubler, and Andrea Buda, "Universal Messaging Standards for the IoT from a Lifecycle Management Perspective" IEEE AUGUST 2014.

[7]. S. Kubler, M. Madhikermi, and K. Fr"amling, "QLM messaging standards: Introduction and comparison with existing messaging protocols," in Service Orientation in Holonic and Multi-Agent Manufacturing and Robotics. New York, NY, USA: Springer, vol. 544, pp. 237-256.

[8]. K. Fr"amling, V. Hinkka, S. Parmar, and J. T"atil"a, "Standards for inter-organizational tracking information exchange in the supply chain information control problems in manufacturing," in Proc. Inf. Control Problems Manuf., Bucharest, Romania, May 2012, vol. 14, pp. 661-666.

[9]. Yi-Bing Lin, Fellow, IEEE, Yun-Wei Lin, Chang-Yen Chih, Tzu-Yi Li, Chia-Chun Tai, Yung-Ching Wang, Fuchun Joseph Lin, Hsien Chung Kuo, Chih-Chieh Huang, Su-Chu Hsu ,"EasyConnect: A Management System for IoT Devices and Its Applications for Interactive Design and Art", JIOT.2015.2423286, IEEE 2015 Internet of Things Journal

[10]. A. P. Castellani, M. Dissegna, N. Bui, and M. Zorzi, "WebIoT: A web application framework for the internet of things," in Proc. IEEE Wireless Commun. Netw. Conf. Workshops, Paris, France, 2012.

[11]. Ing-Ray Chen, Fenye Bao, and Jia Guo "Trust-based Service Management for Social Internet of Things Systems" 1545-5971 (c) 2015 IEEE

[12].Shifeng Fang, Li Da Xu, Yunqiang Zhu, Jiaerheng Ahati, Huan Pei, Jianwu Yan, and Zhihui Liu, "An Integrated System for Regional Environmental Monitoring and Management Based on Internet of Things", IEEE
Transactions On Industrial Informatics, Vol. 10, No. 2, May 2014

[13].Runliang Dou and Guofang Nan, "Optimizing Sensor Network Coverage and Regional Connectivity in Industrial IoT Systems", 1932-8184 @ 2015 IEEE.

[14]. IEEE 802.15 WPAN Task Group 4e (TG4e), IEEE Standard 802.15.4b, 2014.

[15]. L. A. Amaral, F. P. Hessel, E. A. Bezerra, J. C. Corrêa, O. B. Longhi, and T. F. Dias, "eCloudRFID-A mobile software framework architecture for pervasive RFID-based applications," J. Netw. Comput. Appl., vol. 34, no. 3, pp. 972-979, 2011.

[16]. W. He, G. Yan, and L. Xu, "Developing vehicular data cloud services in the IoT environment," IEEE Trans. Ind. Informat., vol. 10, no. 2, pp. 1587-1595, 2014.

[17]. E. Sun, X. Zhang, and Z. Li, "The Internet of Things (IOT) and cloud computing (CC) based tailings dam monitoring and pre-alarm system in mines," Safety Sci., vol. 50, no. 4, pp. 811-815, 2012

[18]. C. Wang, Z. Bi, and L. D. Xu, "IoT and cloud computing in automation of assembly modeling systems," IEEE Trans. Ind. Informat., vol. 10, no. 2, pp. 1426-1434, 2014.

[19]. L. Zhang, Y. Luo, F. Tao, B. Li, L. Ren, X. Zhang, H. Guo, Y. Cheng, A. Hu, and Y. Liu, "Cloud manufacturing: A new manufacturing paradigm," Enterp. Inf. Syst., vol. 8, no. 2, pp. 167-187, Mar. 2014.

[20]. IEEE Communications Society, P1901.2-2013Standard for Low Frequency (less than $500 \mathrm{kHz}$ ) Narrow Band Power Line Communications for Smart Grid Applications, IEEE Standard P1901.2-2013.

[21]. I. Vilajosana, J. Llosa, B. Martinez, M. DomingoPrieto, A. Angles, and X. Vilajosana, "Bootstrapping smart cities through a self-sustainable model based on big data flows," IEEE Commun. Mag., vol. 51, no. 6, pp. 128-134 Jun. 2013

[22]. J. M. Hernández-Muñoz, J. B. Vercher, L. Muñoz, J. A. Galache, M. Presser, L. A. Hernández Gómez, and J. Pettersson, "Smart Cities at the forefront of the future Internet," The Future Internet, Lect. Notes Comput. Sci., vol. 6656, pp. 447-462, 2011.

[23]. C. E. A. Mulligan and M. Olsson, "Architectural implications of smart city business models: An evolutionary perspective," IEEE Commun. Mag., vol. 51, no. 6, pp. 8085, Jun. 2013.

\section{BIOGRAPHIES}

C. S. Patil Currently working towards Masters in Communication Engg from North Maharashtra University, Dhule. Received B.E (Electronics \& Telecom) degree from Pune University, Nasik; 2014. Research interests focuses on Internet of Things

K. N. Pawar Head of Electronics Dept. in SSVPS COE, NMU. His research interest focuses on Smart city and Internet of thing. 